\title{
An Integrated in SEM Multi-Purpose AFM Instrument Utilizing an Active Cantilever
}

Mathias Holz ${ }^{1}$, Alexander Reum², Ahmad Ahmad ${ }^{1,2}$, Tzvetan Ivanov ${ }^{1}$, Christoph Reuter ${ }^{2}$, Martin Hofmann ${ }^{1}$ and Ivo W. Rangelow ${ }^{1}$

1. Nano Analytik GmbH, Ilmenau, Germany.

2. Technische Universität Ilmenau, Ilmenau, Germany.

* Corresponding author: m.holz@nanoanalytik.net

Integration two surface investigation techniques, an atomic force microscopy (AFM) and a scanning electron microscopy (SEM), provides a novel multi-purpose instrument capable in direct visual feedback, correlative microscopy and tip induced fabrication at the nano scale. The invention of selfactuated and self-sensing cantilever (so called active cantilever) [1] make possible the realization of a multipurpose instrument by using specialised tips [2]. The AFM instrument integrated with the SEM (capable of being integrated with all major brands of SEM's) allows accurate correlative measurements easily and quickly with sub-10nm correlation accuracy (Fig.1). Here, we will present AFMfunctionalities combined with high resolution scanning electron microscope and focused ion beam systems for nanoscale characterization. In this context, a six-axis AFM system using active cantilever was developed. SEM viewing angle of $-20^{\circ}$ to $+65^{\circ}$ allows direct control of the AFM-tip position at maintaining a minimum SEM working distance of $4 \mathrm{~mm}$. The design of the developed AFM-integration is described and its performance is demonstrated. The AFM-scanner operate in X,Y,Z in range of $60 \mu \mathrm{m}$ $\times 60 \mu \mathrm{m} \times 20 \mu \mathrm{m}$ where the resolution is $0.2 \mathrm{~nm}$ for $\mathrm{x}$ and $\mathrm{y}$ and $0.1 \mathrm{~nm}$ for $\mathrm{z}$ and allows all common AFM and FIM operation modes. The AFM scanner permits carrying out all measurements at exactly the same point on the sample. Moreover, the functionality of the system can be extended by the use of all active probes in particular: (i) contact/or on-contact AFM, (ii) Current-AFM, Field Emission-Scanning Probe Lithography, (iii) Tip Based-Electron Beam Induced Deposition, (iv) nanomachining, (v) correlative microscopy.

Results from combined examinations applying fast AFM-methods and SEM-image fusion, AFM-SEM combined metrology verification (Fig. 2), and three-dimensional-visualization will be shown. Simultaneous operation of SEM and AFM provides a fast navigation combined with sub-nm topographic image acquisition. Metrology application scenarios of combined studies are discussed, for example of diverse semiconductor structures and devices. We will demonstrate that the combination of active-cantilever based-AFM and conventional SEM accelerates the development of novel correlative methods for nanoscale characterization and nanofabrication.

\section{References:}

[1] IW Rangelow et al., Device and Method for Mask-less AFM Microlithography, U.S. patent 7,141,808 (2005).

[2] IW Rangelow et al., Journal of Vacuum Science \& Technology B, Nanotechnology and Microelectronics: Materials, Processing, Measurement and Phenomena 35 (2017), p. 06G101. doi.org/10.1116/1.4992073

[3] IW Rangelow et al., Journal of Vacuum Science \& Technology B 36 (2018), p. 06J102. doi: $10.116 / 1.5048524$ 


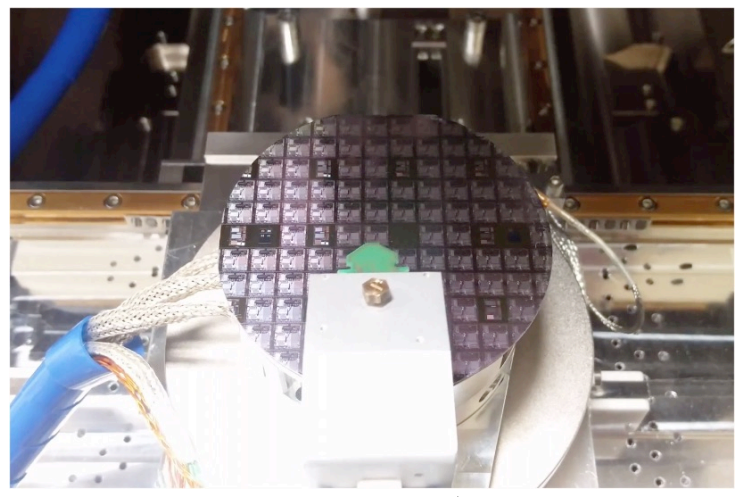

a)

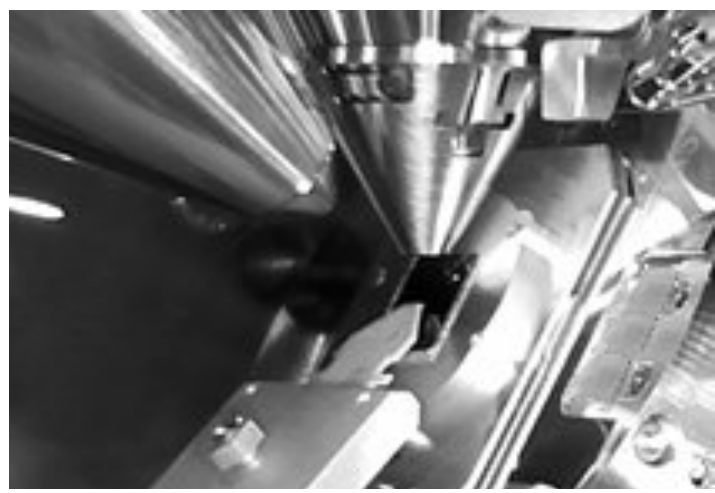

b)

Figure 1. (a) View of the AFM in the opened SEM-chamber; (b) Image of the AFM integrated in the SEM/FIB-tool. The AFM is tilted at $60^{\circ}$ with respect to the SEM column. The tool is combining various patterning and analyzing methods at the same point.
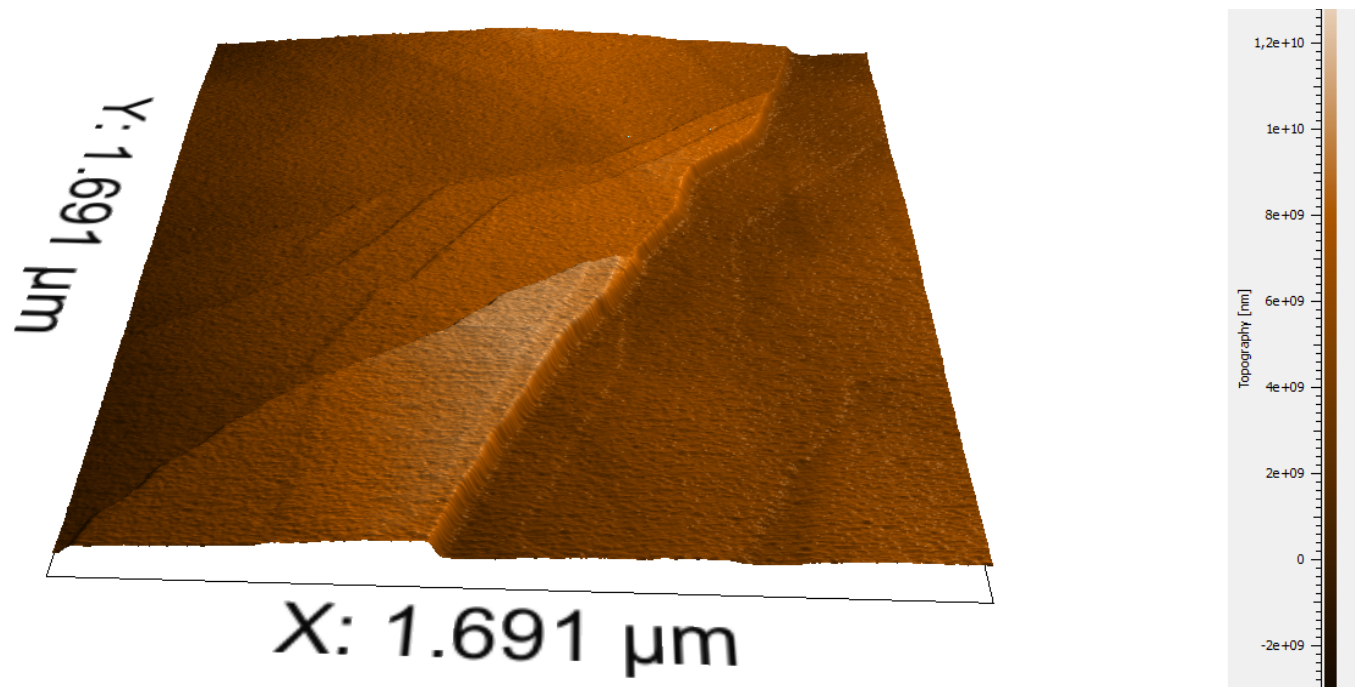

a)
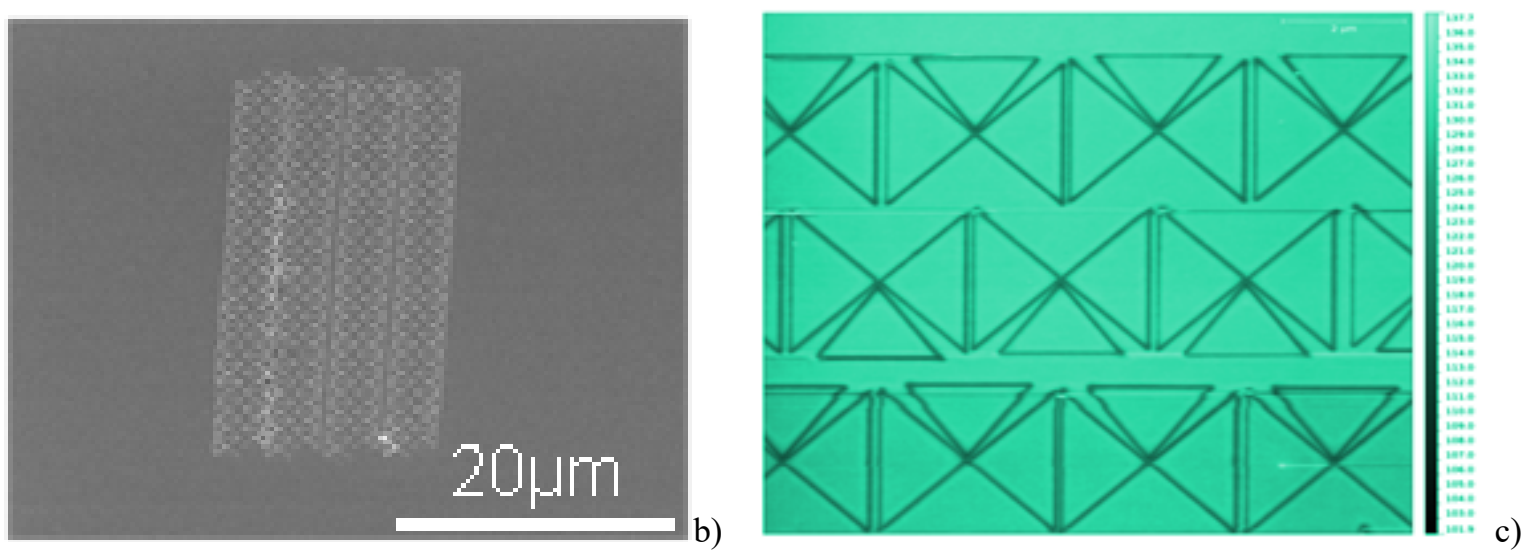

Figure 2. (a) Atomic force microscope (AFM) image of HOPG, indicating single atomic steps obtained by thermomechanically actuated piezoresistive cantilever [1]; (b) SEM-image of Field Emission-Scanning Probe Lithography plasmonic-features realized using a Diamond tip; (c) Non-contact AFM image of the features obtained with the AFMinSEM. 